Mathematical Research Letters 3, 429-437 (1996)

\title{
SQUARE ROOT OF DIVERGENCE OPERATORS, SQUARE FUNCTIONS, AND SINGULAR INTEGRALS
}

\author{
Pascal Auscher and Philippe Tchamitchian
}

A вstract. This letter is to present the main lines of an approach that sheds light on some of the connections between square roots of divergence form differential operators, square functions and Calderón-Zygmund singular integrals.

\section{Introduction}

For almost every $x \in \mathbb{R}^{n}$, let $A(x)$ be a matrix in $M_{n}(\mathbb{C})$, assumed to be uniformly elliptic : there are two constants $C_{0}$ and $\delta>0$ such that $\|A(x)\| \leq C_{0}$ a.e. and

$$
\forall \xi \in \mathbb{C}^{n} \quad \operatorname{Re} A(x) \xi \cdot \bar{\xi} \geq \delta|\xi|^{2} \text {. a.e. }
$$

Following Kato [Ka], let $L=-\operatorname{div}(A \nabla)$ be defined as a maximal accretive operator on $L^{2}\left(\mathbb{R}^{n}\right)$. Such an operator has a holomorphic functional calculus and, in particular, its maximal accretive square root can be defined by, e.g.,

$$
L^{1 / 2} f=\frac{2}{\sqrt{\pi}} \int_{0}^{\infty} e^{-t^{2} L} L f d t, \quad f \in \mathcal{D}(L)
$$

Our work is motivated by Kato' square root problem, which is whether the domain of $L^{1 / 2}$ coincides with $H^{1}\left(\mathbb{R}^{n}\right)$ ? This question reduces to proving the a priori inequality

$$
\left\|L^{1 / 2} f\right\|_{2} \leq c_{1}\|f\|_{H^{1}}, \quad f \in \mathcal{D}(L),
$$

for $L$ and its adjoint, or its homogeneous version

$$
\left\|L^{1 / 2} f\right\|_{2} \leq c\|\nabla f\|_{2}, \quad f \in \mathcal{D}(L) .
$$

Received March 5, 1996. 
In one dimension, these inequalities were obtained by Coifman, McIntosh and Meyer $[\mathrm{CMcM}]$ in 1981. Since then, there has been a considerable interest in finding an $n$-dimensional extension of their results (see [CM], $[\mathrm{Mc} 1],[\mathrm{K}]$ and their references). Our purpose is to describe a possible way of obtaining this extension. Though we have not been completely successful, we do obtain some partial results as well as sharp $L^{p}$ estimates. Our strategy relies on understanding the connections between these problems, singular integrals and square functions.

At this point it is a pleasure to acknowledge helpful discussions with numerous people including G. David, L. Escauriaza, C. Li, A. McIntosh, J. Pipher and to thank Université de Rennes I, Brown University and the ICMS in Edinburgh, where parts of this work have been done. Excerpts of this work were also presented during various lectures including the one delivered by the first author at the workshop in harmonic analysis and partial differential equations at the ICMS in Edinburgh.

\section{Two points of view to attack the problem}

To avoid irrelevant details, we restrict here the discussion to inequality $(\mathrm{K})$. For $t>0$, define $\theta_{t}: L^{2}\left(\mathbb{R}^{n} ; \mathbb{C}^{n}\right) \rightarrow L^{2}\left(\mathbb{R}^{n} ; \mathbb{C}\right)$ by

$$
\theta_{t}=-\frac{2}{\sqrt{\pi}} e^{-t^{2} L} t \operatorname{div} A
$$

where $A$ stands for multiplication by $A(x)$ on $L^{2}\left(\mathbb{R}^{n} ; \mathbb{C}^{n}\right)$ (whose elements are arranged as column vectors).

Lemma 1. (K) is equivalent to the $L^{2}$-boundedness of the "singular integral" $T=\int_{0}^{\infty} \theta_{t} \frac{d t}{t}$.

This is the "singular integral" point of view. We shall see that $T$ is not a Calderón-Zygmund operator in general, but is nevertheless a useful operator in view of $L^{p}$ estimates.

Lemma 2. $(\mathrm{K})$ is equivalent to the $L^{2}$-boundedness of the LittlewoodPaley-Stein functional $g(F)=\left(\int_{0}^{\infty}\left\|\theta_{t} F\right\|_{2}^{2} \frac{d t}{t}\right)^{1 / 2}$, by which we mean $g(F) \leq C\|F\|_{2}$ for all $F \in L^{2}\left(\mathbb{R}^{n} ; C^{n}\right)$.

This is the "square function" point of view, which seems more flexible for proving $(\mathrm{K})$. However, $g$ is not of a classical type.

A key ingredient in the proofs of these lemmata is the topological direct sum

$$
L^{2}\left(\mathbb{R}^{n} ; \mathbb{C}^{n}\right)=\left\{h \in L^{2}\left(\mathbb{R}^{n} ; \mathbb{C}^{n}\right) ; \operatorname{div}(A h)=0 \text { in } \mathcal{D}^{\prime}\left(\mathbb{R}^{n}\right)\right\}+\nabla \dot{H}^{1}\left(\mathbb{R}^{n}\right)
$$


which is a consequence of the ellipticity of $A$. This equality can be thought of an adapted Hodge decomposition for functions in $L^{2}\left(\mathbb{R}^{n} ; \mathbb{C}^{n}\right)$ and is used again later. Other ingredients in the proof of Lemma 2 are results of McIntosh connecting $H^{\infty}$ functional calculi and square function estimates [Mc2].

At this stage, further knowledge on $\theta_{t}$ is needed, which is derived from a natural hypothesis.

\section{The hypothesis $(\mathrm{G})$}

Let $S_{t}(x, y)$ be the distributional kernel of $e^{-t^{2} L}$. We say that $L$ satisfies the hypothesis $(\mathrm{G})$ when $S_{t}(x, y)$ is a Hölder continuous function for each $t>0$ that satisfies

$$
\begin{gathered}
\left|S_{t}(x, y)\right| \leq c t^{-n} e^{-\beta|x-y|^{2} / t^{2}} \\
\left|S_{t}(x, y)-S_{t}(x+h, y)\right|+\left|S_{t}(x, y)-S_{t}(x, y+h)\right| \leq c t^{-n}(|h| / t)^{\mu}
\end{gathered}
$$

for some constants $c, \mu>0$ et $\beta>0$.

These estimates go back to Aronson and Nash ([Ar], [Na]) when $A$ is real valued. In the complex case, here is a summary of works by Coulhon, Escauriaza, McIntosh and the authors ([A], $[\mathrm{ACT}],[\mathrm{AE}],[\mathrm{AMcT}])$.

\section{Theorem 1.}

(i) (G) holds if $n=1$ or $n=2$;

(ii) (G) holds if the entries of $A$ are uniformly continuous or in vmo when $n \geq 3$ (up to a technical change in (4));

(iii) (G) is stable under $L^{\infty}$ perturbation of the entries of $A$;

(iv) if $n \geq 5$, there are counterexamples to $(\mathrm{G})$.

By using parabolic Cacciopoli's inequalities we can deduce the following estimates on $\theta_{t}(x, y)$, the kernel of $\theta_{t}$.

Lemma 3. Under the hypothesis $(\mathrm{G})$, there are constants $c, \alpha>0$ and $\eta>0$ such that for every $t>0, r>0, x, h \in \mathbb{R}^{n}$ with $2|h| \leq t+r$,

$$
\int_{r \leq|x-y| \leq 2 r}\left|\theta_{t}(x, y)\right| d y \leq c\left(\frac{r}{t}\right)^{n-1} e^{-\alpha r^{2} / t^{2}}
$$

$$
\int_{r \leq|x-y| \leq 2 r}\left|\theta_{t}(x+h, y)-\theta_{t}(x, y)\right| d y \leq c\left(\frac{|h|}{t+r}\right)^{\eta}\left(\frac{r}{t}\right)^{n-1} e^{-\alpha r^{2} / t^{2}}
$$


The main source of difficulties is the absence of regularity in the $y$ variable in general as simple examples show. Nevertheless, a special form of regularity occurs. Indeed,

$$
\left\|\theta_{t} \nabla f\right\|_{2} \leq \frac{c}{t}\|f\|_{2}, \quad f \in \mathcal{D}(L)
$$

which follows from the identity $\theta_{t} \nabla f=-\frac{1}{\sqrt{\pi}} \frac{d}{d t}\left(e^{-t^{2} L} f\right)$, together with holomorphic functional calculus. In fact, $t \operatorname{div}_{y} \theta_{t}(x, y)$, the kernel of $t \theta_{t} \nabla$, is a function dominated pointwise by a gaussian.

\section{Square function and Carleson measure estimates}

Let $\left(e_{j}\right)_{1 \leq j \leq n}$ be the canonical basis of $\mathbb{C}^{n}$. Denote by $\left|\theta_{t}(I)\right|_{c}$ the smallest constant $\bar{C} \geq 0$ (possibly infinite) in the following Carleson measure estimate

$$
\forall B \in \mathcal{B} \quad \int_{B} \int_{0}^{r(B)} \sum_{j=1}^{n}\left|\theta_{t}\left(e_{j}\right)(x)\right|^{2} \frac{d x d t}{t} \leq C^{2}|B|,
$$

where $\mathcal{B}$ denotes the collection of all Euclidean balls $B$ and $r(B)$ the radius of $B$. One interesting feature in this quantity is the local nature of the left-hand side in $(7)$.

Let $\left|\theta_{t}\right|_{\mathcal{S}}=\sup \left\{g(F) ;\|F\|_{2} \leq 1\right\}$, where $g$ is defined in Lemma 2 .

A natural strategy from harmonic analysis is to relate the finiteness of $\left|\theta_{t}\right|_{\mathcal{S}}$ to a Carleson measure estimate, i.e., $\left|\theta_{t}(I)\right|_{c}$ finite. Indeed, had $\theta_{t}(x, y)$ been Hölder continuous in $y$, even in some averaged sense, then, using also the decay estimates (5a), a variant of Christ and Journé's Theorem 1 in [CJ] would suffice. This is not the case here, but the adapted Hodge decomposition is used again to get round the absence of regularity of $\theta_{t}(x, y)$ and to take advantage of (6).

Theorem 2. Assume that $(\mathrm{G})$ holds. Then $(\mathrm{K})$ is equivalent to the finiteness of $\left|\theta_{t}(I)\right|_{c}$. More precisely, there are constants $c_{i}$ depending only on $n, \delta$ and $C_{0}$ such that if $\left|\theta_{t}\right|_{w}$ denotes the smallest constant $c$ in both (5a) and (6),

$$
\left|\theta_{t}\right|_{\mathcal{S}} \leq c_{1}\left|\theta_{t}(I)\right|_{c}+c_{2}\left|\theta_{t}\right|_{w} \quad \text { and } \quad\left|\theta_{t}(I)\right|_{c} \leq c_{3}\left|\theta_{t}\right|_{\mathcal{S}}+c_{4}\left|\theta_{t}\right|_{w}
$$

Actually, this theorem is a particular case of a general result for characterizing the $L^{2}$-boundedness of a class of square functions (see [AT3]).

In order to compute $\left|\theta_{t}(I)\right|_{c}$, we introduce an estimator that is related to the solutions of a family of Dirichlet problems for $L$. For each ball $B \in \mathcal{B}$, 
let $\tilde{B}$ be the ball with same center and twice its radius and, for $1 \leq j \leq n$, define $f_{j, B} \in H_{0}^{1}(\tilde{B})$ as the unique solution of the equation

$$
\operatorname{div}\left(A \nabla f_{j, B}\right)=\operatorname{div}\left(A e_{j}\right) \quad \text { on } \tilde{B} .
$$

Calculations using the estimate of Lemma 3 show that

(9) $\int_{B} \int_{0}^{r(B)}\left|\theta_{t}\left(e_{j}\right)(x)\right|^{2} \frac{d x d t}{t}=\int_{B} \int_{0}^{r(B)}\left|\theta_{t}\left(\nabla f_{j, B}\right)(x)\right|^{2} \frac{d x d t}{t}+O(|B|)$.

When $A$ is real and symmetric, integrations by parts starting from (9) lead to $\left|\theta_{t}(I)\right|_{c}<\infty$, which gives a constructive proof of $(\mathrm{K})$. In this case, $(\mathrm{K})$ can also be proved by applying boundary Rellich identities to the solutions of the elliptic equation $\partial^{2} u / \partial_{t}^{2}+\operatorname{div}(A \nabla u)=0$ on $\mathbb{R}^{n} \times(0, \infty)$ as in $[\mathrm{KP}]$. Our approach may be seen as the Carleson measure version (in the parabolic setting but there is an elliptic analog) of Rellich inequalities. Let us also mention that $\left|\theta_{t}(I)\right|_{c}<\infty$ implies refinement over the moment estimates established by Nash [Na].

The next result is of a perturbation type, using (8), (9) and the estimator

$$
\gamma(L)=\sup _{B \in \mathcal{B}}\left(\frac{1}{|\tilde{B}|} \int_{\tilde{B}} \sum_{j=1}^{n}\left|\nabla f_{j, B}\right|^{2}\right)^{1 / 2} .
$$

Theorem 3. There is an $\varepsilon=\varepsilon\left(n, \delta, C_{0}\right)>0$ such that $(\mathrm{K})$ holds provided $\gamma(L)<\varepsilon$.

It is easy to see that $\gamma(L) \leq C(n) \delta^{-1}\|A\|_{B M O}$ where $\delta$ is the best constant in (1). Hence we can deduce the validity of Kato's conjecture in all cases previously known.

Corollary 4. There are $\varepsilon_{i}=\varepsilon_{i}\left(n, \delta, C_{0}\right)>0$ such that $(\mathrm{K})$ holds whenever either (i) $\|I-A\|_{\infty}<\varepsilon_{1}$ or (ii) $\|A\|_{B M O}<\varepsilon_{2}$.

This result is proved in $[\mathrm{CDM}]$ and [FJK1] in case (i) and in $[\mathrm{E}]$ in case (ii). We also show that it is possible to take $\varepsilon_{1}=(1+4 n)^{-1 / 2}$, which slighlty improves over Journé's constant in $[\mathrm{J}]$. Multilinear expansions are used in these works.

There is a local version of Theorem 3, where the local estimator is defined by taking the supremum over small balls only. This proves $(K)^{\prime}$ when $A$ is uniformly continuous ([FJK2], unpublished) or when $A \in v m o$ ([E], unpublished). Here, we also show that these results are stable by perturbation of the coefficients in either $L^{\infty}$ or $B M O$. 
An important remark at this stage is that we did not assumed $(G)$ in Theorem 3. The reason is that, thanks to an abstract result in [AMcN1], the functional $g$ of Lemma 2 can be replaced by other functionals to which, without any restriction on the matrix A, our general result on characterizing $L^{2}$ boundedness of square functions can be applied. Eventually, the estimators used for the new functionals are the same.

Variations of the preceeding methods give us the following result, whose hypothesis is not comparable to that of the local version of Theorem 3 .

We say that a function $f$ in $B M O$ is Carleson regular locally if there are a non decreasing weight $w:[1, \infty) \rightarrow[1, \infty)$ such that $\lim _{t \rightarrow \infty} w(t)=\infty$ and a constant $C \geq 0$ for which

$$
\forall B \in \mathcal{B}, r(B) \leq 1, \quad \int_{B} \int_{0}^{r(B)}\left|\Delta_{t} f(x)\right|^{2} w\left(\frac{r(B)}{t}\right) \frac{d x d t}{t} \leq C|B|,
$$

where $\Delta_{t} f=f * \Delta_{t}$ is a Littlewood-Paley decomposition of $f$. Of course, this condition does not depend on the particular choice of $\Delta_{t}$.

Theorem 5. Assume that the coefficients of $A$ are Carleson regular locally. Then $(K)^{\prime}$ is valid.

Condition (10) is a local regularity condition, satisfied by functions in $v m o$ or in $H_{\text {loc,unif }}^{s}\left(\mathbb{R}^{n}\right)$ for any $s>0$. This result, therefore, generalizes a theorem in [Mc3]. For instance, the characteristic function of any Lipschitz domain or of any planar set whose boundary is a snowflake is Carleson regular locally. Wildely oscillating functions such as $\sin \frac{1}{x}$ in dimension 1 are not.

\section{Singular integrals and $L^{p}$ estimates}

We return to the singular integral point of view. Here, the hypothesis (G) plays an important role.

In dimension 1, it is shown in [AT1] that the operator $T$, as defined in Lemma 1, is a Calderón-Zygmund operator. This is no longer true when $n \geq 2$ as observed by C. Kenig, whom we gratefully thank for having communicated to us the following unpublished counterexample.

Lemma 4. For every $\varepsilon>0$ there is a real self-adjoint operator $L=-\operatorname{div}(A \nabla)$ on $L^{2}\left(\mathbb{R}^{2}\right)$ for which the a priori inequality

$$
\|\nabla f\|_{p} \leq c_{p}\left\|L^{1 / 2} f\right\|_{p}, \quad f \in \mathcal{D}(L),
$$

fails when $p \geq 2+\varepsilon$.

This lemma implies that $T$ is not bounded on $L^{p}$ when $p \leq 2-\varepsilon /(1+\varepsilon)$ although it is bounded on $L^{2}$. In particular, $T$ does not have a CalderónZygmund kernel. Nevertheless, some estimates are available, including this consequence of $(5 b)$. 
Lemma 5. Under the hypothesis $(\mathrm{G})$, the kernel $T(x, y)$ of $T$ satisfies the Hörmander condition

$$
\sup _{x, h \in \mathbb{R}^{n}} \int_{|x-y| \geq 2|h|}|T(x+h, y)-T(x, y)| d y<\infty .
$$

Furthermore, the factorization $L^{1 / 2}=T \nabla$ is not unique in the sense that $L^{1 / 2}=U \nabla$ for any $U$ such that $(U-T) \nabla=0$. Taking advantage of this freedom and using smoothing procedures on the kernel of $\theta_{t}$ together with Lemma 1 and Lemma 5, we have

Theorem 6. If $(\mathrm{G})$ holds, then there is a singular integral operator $U$ with Calderón-Zygmund kernel such that

$$
L^{1 / 2} f=U \nabla f, \quad f \in \mathcal{D}(L) .
$$

Moreover, $(\mathrm{K})$ holds if and only if $U$ is $L^{2}$-bounded.

From Calderón-Zygmund theory and an analytic perturbation argument we get

Theorem 7. Let $n \geq 2$. If the hypothesis $(\mathrm{G})$ and the equivalence $\left\|L^{1 / 2} f\right\|_{2} \sim\|\nabla f\|_{2}$ hold then there is an $\varepsilon=\varepsilon\left(n, \delta, C_{0}\right)>0$ for which the following a priori inequalities hold when $f \in \mathcal{D}(L)$ :

$$
\begin{gathered}
\left\|L^{1 / 2} f\right\|_{p} \leq c_{p}\|\nabla f\|_{p}, \quad 1<p<\infty \\
\|\nabla f\|_{p} \leq c_{p}\left\|L^{1 / 2} f\right\|_{p}, \quad 1<p<2+\varepsilon,
\end{gathered}
$$

Using a slightly strengthened version of (11) we also obtain the endpoint estimates:

$$
\left\|L^{1 / 2} f\right\|_{\mathcal{H}^{1}\left(\mathbb{R}^{n}\right)} \sim\|\nabla f\|_{\mathcal{H}^{1}\left(\mathbb{R}^{n}\right)},
$$

where $\mathcal{H}^{1}\left(\mathbb{R}^{n}\right)$ is the Hardy space of Stein and Weiss, and

$$
\left\|L^{1 / 2} f\right\|_{B M O} \leq c\|A \nabla f\|_{B M O} .
$$

In view of Lemma 4 , the ranges for $p$ are best possible. Note that the $L^{\infty}-B M O$ boundedness of $T$ is essential in the proof of the last statement in Theorem 6 .

Theorem 7 applies, e.g., when $A$ is real and symmetric. In this case, (13) in the range $(1,2+\varepsilon)$ and the $p=1$ endpoint result may be compared to results in $[\mathrm{KP}]$ where the situation is slightly different. Indeed, these estimates can be rephrased in terms of solvability results in $L^{p}$ space of the regularity and Neumann problems for the elliptic equation $\partial^{2} u / \partial_{t}^{2}+$ $\operatorname{div}(A \nabla u)=0$ on $\mathbb{R}^{n} \times(0, \infty)$ (see $[\mathrm{K}]$ for an account on this topic when $p=2)$. 


\section{Further results}

a) Further elaborations on the techniques presented above give us other types of new results. One sample is

Theorem 8. $(\mathrm{K})$ is valid when $A=B+M$, where $\|M\|_{\infty}<\varepsilon(B, n)$ and $B$ depends only on one variable. Moreover, when $M=0$, (13) is valid in the full range $1<p<\infty$.

b) Bounded domains.

Let $\Omega$ be a bounded Lipschitz domain in $\mathbb{R}^{n}$. Let $A(x) \in M_{n}(\mathbb{C})$ be uniformly elliptic in $\Omega$ in the sense of (1) and construct $L=-\operatorname{div}(A \nabla)$ on $\Omega$ with Dirichlet boundary conditions. The square root problem for $L$ is concerned with determining whether $\mathcal{D}\left(L^{1 / 2}\right)=H_{0}^{1}(\Omega)$.

When $n=1$, this question is solved in the affirmative (with arbitrary boundary conditions) in [AT2]. Another argument is in [AMcN2]. When $n \geq 2$, the best results known so far seem to be those of McIntosh in [Mc3] who requires some smoothness on the coefficients of $A$.

The points of view developed above are flexible enough to be adapted to this situation. In particular, we obtain analogs of Theorems 3 and 7 . There, the collection $\mathcal{B}$ is replaced by the collection of sets $B \cap \Omega$, where $B \in \mathcal{B}, r(B) \leq 2 \operatorname{diam}(\Omega)$, and with center in $\Omega$. We also make use of Calderón-Zygmund theory since $(\Omega,||, d x)$ is a space of homogeneous type. For example, $\mathcal{D}\left(L^{1 / 2}\right)=H_{0}^{1}(\Omega)$ when $A$ is a perturbation of the identity matrix or when $A$ is uniformly continuous on $\bar{\Omega}$.

It is not clear at this time how to handle other boundary conditions.

\section{References}

[Ar] D. Aronson, Bounds for fundamental solutions of a parabolic equation, Bull. Amer. Math. Soc. 73 (1967), 890-896.

[A] P. Auscher, Regularity theorems and heat kernels for elliptic operators, to appear in J. London Math. Soc.

[AMcT] P. Auscher, T. Coulhon, and Ph. Tchamitchian, Absence de principe du maximum pour certaines équations paraboliques complexes., to appear in Colloq. Math..

[AE] P. Auscher and L. Escauriaza, oral communication.

[AMcN1] P. Auscher, A. McIntosh, and A. Nahmod, Holomorphic functional calculi of operators, quadratic estimates and interpolation, preprint.

[AMcN2] _ The square root problem of Kato in one dimension, and first order elliptic systems, preprint.

[AMcT] P. Auscher, A. McIntosh, and Ph. Tchamitchian, Noyau de la chaleur d'opérateurs elliptiques complexes, Math. Res. Lett. 1 (1994), 37-45.

[AT1] P. Auscher and Ph. Tchamitchian, Calcul fonctionnel précisé pour des opérateurs différentiels complexes en dimension 1 et équations elliptiques complexes en dimension 2, Ann. Inst. Fourier 45 (1995), 721-778. 
[AT2] Conjecture de Kato sur des ouverts de $\mathbb{R}$, Rev. Mat. Iberoamericana 8 (1992), 149-201.

[AT3] $\quad$ in preparation.

[CJ] M. Christ and J.-L. Journé, Polynomial growth estimates for multilinear singular integral operators, Acta Math. 159 (1987), 51-80.

[CDM] R. Coifman, D.G. Deng, and Y. Meyer, Domaine de la racine carrée de certains opérateurs différentiels accrétifs, Ann. Inst. Fourier 33 (1983), 123-134.

[CMcM] R. Coifman, A. McIntosh, and Y. Meyer, L'intégrale de Cauchy définit un opérateur borné sur $L^{2}(\mathbf{R})$ pour les courbes lipschitziennes, Ann. of Math. 116, 361-387.

[CM] R. Coifman and Y. Meyer, Ondelettes et Opérateurs, tome III, Hermann, 1991.

[E] L. Escauriaza, unpublished.

[FJK1] E. B. Fabes, D. S. Jerison, and C. E. Kenig, Multilinear Littlewood-Paley estimates with applications to partial differential equations, Proc. Nat. Acad. Sci. U.S.A. 79 (1982), 5746-5750.

[FJK2] unpublished.

[J] J.-L. Journé, Remarks on the square root problem, Publ. Math. 35 (1991), 299-321.

[Ka] T. Kato, Perturbation theory for linear operators, Springer-Verlag, New York, 1966.

[K] C. E. Kenig, Harmonic Analysis techniques for second order elliptic boundary value problems, CBMS no 83, NSF Regional Conferences in Mathematics (1994).

[KP] P. Kenig and J. Pipher, The Neumann problem for elliptic equations with non-smooth coefficients, Invent. Math. 113 (1993), 447-509.

[Mc1] A. McIntosh, The square root problem for elliptic operators, in Functional analytic methods for partial differential equations, Lecture Notes in Math., Springer Verlag, Berlin, 1450 (1990), 122-140.

[Mc2] Operators which have an $H^{\infty}$ functional calculus, Miniconference on operator theory and partial differential equations, Proc. Centre Math. Analysis, Austral Nat. Univ. 14 (1986), 210-231.

[Mc3] , Square roots of elliptic operators, J. Funct. Anal. 61 (1985), 307-327.

IR M A R, Université De Rennes I, Campus de Beaulieu, F-35042 Rennes Cedex and Brown University, Providence, RI 02912 USA

Current address: LAMFA, faculté de mathématique et d'informatique, Université de Picardie-Jules Verne, 33, rue Saint Leu, F-80039 Amiens Cedex 1

E-mail address: auscher@mathinfo.u-picardie.fr

FaCulté des SCiences et Techniques de Saint Jérôme, Univerité D'A ixMarseille Iit, Avenue Escadrille Normandie-Niemen, F-13397 Marseille CEDEX 20 AND LATP, CNRS, URA 225

E-mail address: tchamphi@vmesa11.u-3mrs.fr 